\title{
Computational Identification, Characterization and Analysis of Conserved miRNAs and their Targets in Amborella Trichopoda
}

Hajieghrari $\mathbf{B}^{1,2}$, Farrokhi $\mathbf{N}^{3^{*}}$, Goliaei $\mathbf{B}^{4^{*}}$ and Kavousi $\mathrm{K}^{1}$

${ }^{1}$ Department of Bioinformatics, Institute of Biochemistry and Biophysics (IBB), University of Tehran, Tehran, Iran

${ }^{2}$ Department of Agricultural Biotechnology, College of Agriculture, University of Jahrom, Jahrom, Iran

${ }^{3}$ Department of Agronomy and Plant Breeding/ Biotechnology Research Group, Faculty of Agriculture, Shahrood University, Shahrood, Iran

${ }^{4}$ Laboratory of Complex Biological Systems and Bioinformatics (CBB), Institute of Biochemistry and Biophysics (IBB), University of Tehran, Tehran, Iran

\begin{abstract}
MicroRNAs (miRNAs) are single stranded non-coding endogenous small RNAs of about 22 nucleotides, which are directly involved in regulating gene expression at post transcriptional level. miRNAs play key roles in development and response to biotic and abiotic stresses. Homology searches allow identification of new miRNAs due to their relative high conservation in plant species. Here, miRNAs were identified for Amborella trichopoda. Known and unique plant miRNAs from miRBase were BLAST-searched against Expressed Sequence Tag (EST) and Genomic Survey Sequence (GSS) in $A$. trichopoda. All candidate sequences with appropriate fold back structures were screened by a series of miRNA filtering criteria. Finally, we identified and analysed conservation of 5 potential conserved miRNAs belonging to 5 miRNA gene families from ESTs as well as 82 newly identified miRNAs belonging to 39 miRNA families from GSSs. Potential target genes of identified miRNAs were identified based on their sequence complementarities to the respective miRNAs using psRNATarget against scaffold assignment of $A$. trichopoda genome sequences. Totally, 1219 target sites in $A$. trichopoda genome were identified. From which, $941(77.19 \%)$ were predicted to be the subject of miRNA cleavage and $278(22.81 \%)$ scaffolds were regulated via translational repression of mRNA. From the predicted miRNAs, 18 had no target sequence in A.trichopoda.
\end{abstract}

Keywords: Micro RNA (miRNA); Amborella trichopoda; Homology search; Target genes

\section{Introduction}

Micro RNAs (miRNAs) are a class of endogenous, single-stranded, non-protein-coding small RNAs that negatively regulate expression of varieties of protein-coding genes at post transcriptional level. This ancient evolutionary mechanism controls the expression by both targeting and cleavage of complementary mRNA, or in some cases by translational repression [1].

In animals miRNA genes are derived from introns, untranslated regions of transcripts or primary transcripts containing tandem precursors [2]. The animal miRNAs have multiple complementarily recognition sites, an imperfect complementarity that is located at 3'-untranslated region (3'UTR) of their targets. In plants, miRNA genes usually exist as independent transcriptional units and are transcribed by RNA polymerase II into long primary transcripts (Pri-miRNA) [3] with a sole and specific target site. The transcripts from miRNA genes are capped by adding a 5'-7-methylguanosine cap [4], spliced and polyadenylated at $3^{\prime}$ end $[5,6]$. In plants, maturation of miRNA from pre-miRNA is processed in the nucleus by miRNA processing machinery. RNase III like protein DCL1 (Dicer like 1 enzyme), the core component of miRNA processing machinery, shapes the pri-miRNA into the precursor miRNA (pre-miRNA; the hairpin form of primiRNA) within the cytoplasm. The precursor miRNA folds back on itself to form a hairpin secondary structure. Mature miRNA sequences are located on one arm of this hairpin structure. Several paralogs of Dicer-like proteins are present in plants but only DCL1 incorporates in pre-miRNA processing. Other family members have evolved to protect plants against viruses [7]. The pre-miRNA is subsequently cleaved into a 22 bp double-stranded RNA. One strand is the mature miRNA, while the other comes from the opposite arm of the hairpin known as the miRNA* $^{*}$ [8]. Similar to other types of RNA molecules processed by RNase III family of enzymes, miRNA duplex bears two protruding nucleotides at $3^{\prime}$ end [9]. Subsequently, the miRNA/miRNA* duplex is methylated by methyltransferase activity [9]. The processed 2'-O-methylated miRNA/miRNA* may be exported from nucleus to cytoplasm. This transport can be carried out either by HASTY (the Exportin 5 homolog in plants) -dependant or HASTY-independent nucleo cytoplasmic pathways resided on nucleus membrane [10-12]. Finally, the single-stranded mature miRNA is assembled into the ARGONAUTE 1 (Ago1) associated RNA-induced silencing complex (RISC) [13]. This complex is capable of binding to the complementary sequence of an mRNA molecule; the binding can be partial or complete. This results in either mRNA cleavage or translation arrest by miRNA, while the miRNA* is degraded. A given miRNA may have hundreds of different mRNA targets and it may cleave and/or repress the production of hundreds of proteins. An mRNA target can also be regulated by multiple miRNAs [14].

In animals, miRNA sequences have multiple complementarily recognition sites, located at 3'-untranslated region (3'UTR) of their targets. In plants, target mRNAs most contain one continuous complementary recognition site resulting in cleaving the target mRNA

*Corresponding author: Goliaei B, Departments of Biophysics and Bioinformatics laboratories, Institute of Biochemistry and Biophysics (IBB), University of Tehran Tehran, Iran, Tel: +98-216111-3356; E-mail: goliaei@ibb.ut.ac.ir

Farrokhi N, Department of Agronomy and Plant Breeding/ Biotechnology Research Group, Faculty of Agriculture, Shahrood University, Shahrood, Iran Tel: 0098-21-44580; E-mail: nfarrokh@nigeb.ac.ir

Received December 02, 2014; Accepted February 03, 2015; Published February 10, 2015

Citation: Hajieghrari B, Farrokhi N, Goliaei B, Kavousi K (2015) Computationa Identification, Characterization and Analysis of Conserved miRNAs and thei Targets in Amborella Trichopoda. J Data Mining Genomics Proteomics 6: 168 doi:10.4172/2153-0602.1000168

Copyright: (C) 2015 Hajieghrari B, et al. This is an open-access article distributed under the terms of the Creative Commons Attribution License, which permits unrestricted use, distribution, and reproduction in any medium, provided the original author and source are credited. 
Citation: Hajieghrari B, Farrokhi N, Goliaei B, Kavousi K (2015) Computational Identification, Characterization and Analysis of Conserved miRNAs and their Targets in Amborella Trichopoda. J Data Mining Genomics Proteomics 6: 168. doi:10.4172/2153-0602.1000168

and causing an immediate degradation. Additionally and less often, in plants, target site can be found in non-coding region (3'UTR or 5'UTR) of the transcript [15].

In recent years extensive research efforts have focused on identification of potential miRNAs in plant species. Both experimental cloning (construction and sequencing of small RNA libraries) and computational approaches have been used to identify plant miRNAs. Cloning or deep sequencing is limited to finding miRNAs by highly constrained tissue- and time-specific expression patterns. Since many miRNAs are highly conserved amongst species, homology-based search methods may be a useful task towards discovery of new counterparts with the capability of folding into hairpin secondary structures [16]. Additional criteria are necessary to set for distinguishing miRNA from other types of small RNA, reducing the number of false positives. Minimal folding free energy (MFE) and minimal folding free energy index (MFEI) are amongst such criteria; miRNAs have significantly higher negative MFE and MFEI than other RNA types [17,18].

A common approach for the identification of orthologs of miRNAs in other plant species would be through homology search in EST and GSS databases [19], especially for the species whose genomes are unknown or poorly understood. It has been generally accepted that mature miRNAs are conserved in plants from species to species in contrast to animals that miRNA precursors are usually conserved $[5,20]$. This feature of evolutionary conservation of miRNA allows for comparative analysis by using the available bioinformatics tools to search for putative miRNAs. Although large numbers of miRNAs have been identified in plants via this approach [21-25], less-conserved miRNA usually remained unidentified. Nevertheless, predicted miRNAs are needed to be experimentally characterized.

Amborella is a monotypic genus of rare understory shrubs or small trees endemic to the main island, Grande Terre, of New Caledonia.
The genus is placed alone in the family Amborellaceae and contains a single species, Amborella trichopoda. A. trichopoda has pivotal phylogenetic position since it has common ancestor with all other extinct angiosperms.To date, totally 124 A.trichopoda miRNA have been identified [26] and deposited at the miRNA database current version (release 21; June 2014). Due to the importance of $A$. trichopoda as the basal lineage in angiosperms clade in the evolutionary analysis, we applied an EST and GSS based-homology search to identify its potential miRNAs.

\section{Materials and Methods}

\section{Data sets and software}

All 7057 mature miRNA sequences of 73 plant species were downloaded from the miRNA registry miRBase (http://www.mirbase. org; release 21, June 2014) [27-29]. The repeated miRNA sequences were removed from the data set and only the unique ones were used as the reference set. A.trichopoda ESTs and GSSs were obtained from the relevant databases, which were available at nucleotide database of the National Center for Biotechnology Information (NCBI) (http://www. ncbi.nlm.nih.gov). BLAST-2.2.22 [30] was downloaded from NCBI website and set up locally. For prediction of the secondary structure of pre-miRNA and the free energy, Zuker RNA folding algorithm MFOLD 3.5 (http://www.mfold.rna.albany.edu ) was used [31]. The miRNA target genes were predicted by the plant small RNA analysis server psRNATarget (http://www.bioinfo3.noble.org/psRNATarget) [32] and the plant Ensemble database (http://plants.ensembl.org).

\section{Procedure and screening criteria for miRNA prediction}

The workflow for prediction of the potential miRNAs is shown in Figure 1. After removing redundant sequences, all mature plant miRNA sequences downloaded from miRNA registry miRBase were

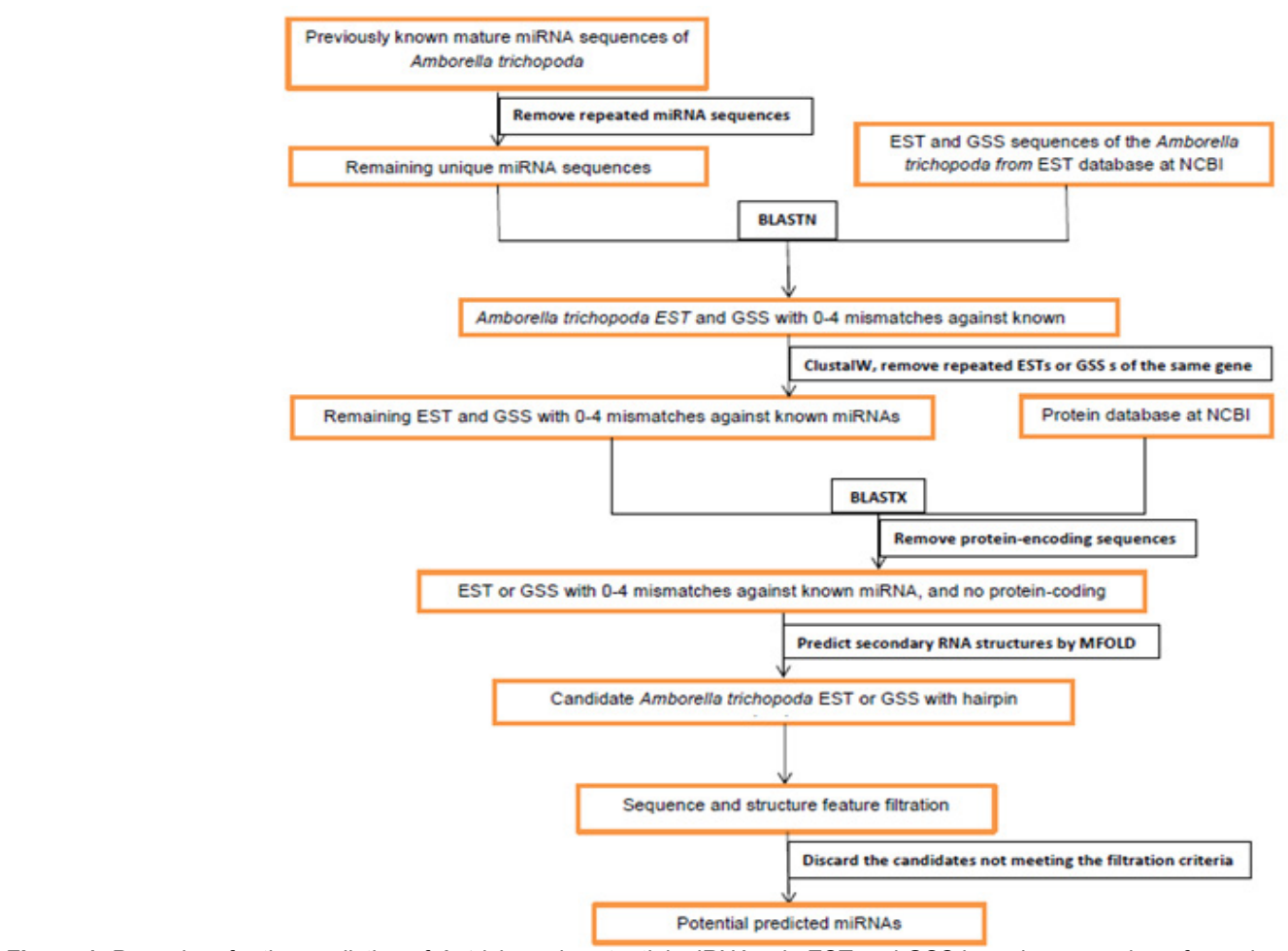

Figure 1: Procedure for the prediction of $A$. trichopoda potential miRNAs via EST and GSS homologs search performed on previously reported plant miRNAs. 
Citation: Hajieghrari B, Farrokhi N, Goliaei B, Kavousi K (2015) Computational Identification, Characterization and Analysis of Conserved miRNAs and their Targets in Amborella Trichopoda. J Data Mining Genomics Proteomics 6: 168. doi:10.4172/2153-0602.1000168

used as queries in BLAST homology search against downloaded ESTs and GSSs. The default settings of BLAST parameters were used, except for maximum target sequence and expected threshold that were set to 1000 and 10, respectively. All ESTs and GSSs with no more than 4 mismatches were selected. The protein-coding sequences were removed from extracted sequences by searching against NCBI nonredundant (nr) protein databases using BLASTX (http://www.BLAST. ncbi.nlm.nih.gov/BLAST.cgi). The precursor sequence of $400 \mathrm{nt}$ was extracted from each sequence by selecting $200 \mathrm{nt}$ upstream and $200 \mathrm{nt}$ downstream of each BLAST hit. If the length of the query was shorter than $400 \mathrm{nt}$, the entire sequence was used as a putative miRNA precursor. The secondary structures of putative pri-miRNAs were predicted using MFOLD 3.5 program. All parameters were set to default values. The $\mathrm{A}+\mathrm{U} / \mathrm{T}$ and $\mathrm{C}+\mathrm{G}$ content and minimal free folding energy index (MFEI) were calculated according to [17] and based on the following equation: $\left.\mathrm{MFEI}=\left[(\mathrm{MFE} / \text { length of the RNA sequence })^{\star} 100\right] /(\mathrm{G}+\mathrm{C}) \%\right]$. The sequences were considered as potential miRNA candidates if they met the following criteria: 1) Mature miRNA should be 18-22 nt in length. 2) The predicted pre-miRNA sequence folded into perfect or nearly perfect stem-loop hairpin secondary structure. 3) The potential mature miRNA sequence located on one arm of the hairpin structure. 4) No loops or breaks were allowed in the miRNA/miRNA* duplex. 5) Predicted mature miRNA sequence had less than 4 nt impairs with the miRNA* sequence. 6) No loops or breaks in miRNA* and miRNA sequences. 7) $\mathrm{A}+\mathrm{U}$ content should be $30-75 \%$. 8) The predicted premiRNA secondary structure had a high negative MFE (lower than $-20 \mathrm{kcal} / \mathrm{mol}$ ) [22] and high MFEI values usually over 0.8 .

\section{Phylogenetic analysis of the miRNAs}

Most plant miRNA and pre-miRNA are strongly conserved with high sequence identity even between distantly related species [20,33], belonging to the same families with low rate of evolution. Therefore, multiple sequence alignment of consensus structure of precursor sequences of the newly identified miRNAs with all previously verified members of each predicted miRNA family in the plant species (obtained from the miRNA database: http://www.mirbase.org/)(release 21, June 2014) [27-29] was constructed by the web based software Loc ARNA (http://rna.informatik.uni-freiburg.de/LocARNA/Input.jsp) [34]. Thus, to obtain further insights on the evolutionary relationships of the newly identified miRNAs and their counterparts in other plant species, phylogenetic trees of the aligned sequences were constructed using average percentage identity. Moreover, to obtain further insights on the evolutionary relationships of the newly predicted conserved miRNAs and their counterparts in other plant species, phylogenetic trees of predicted pre-miRNA sequences were constructed based on average distance using percent identity in CLUSTALW (available online in EMBL/EBI; http://www.ebi.ac.uk/Tools/msa/clustalw2) and the trees were generated in Jalview 2.8.2.

\section{Prediction of potential target genes in Amborella trichopoda}

Predicted miRNAs were used as query against scaffold assignment of Amborella trichopoda draft genome sequence gene index using psRNA Target [32], an updated version of web-based miRU [6]. psRNA Target tool provides reverse complementary matching between miRNAs and their target transcripts and finds target site accessibility by calculating unpaired energy (UPE) necessary for opening the secondary structure around the miRNA target site [32] with the following criteria: 1) No gaps and no more than 4 mismatches are allowed between the mature miRNA and its potential target(s). 2) No mismatch was allowed between position $10^{\text {th }}$ and $11^{\text {th }} .3$ ) No more than one mismatch was allowed at nucleotide positions $2^{\text {nd }}-12^{\text {th }}$ and up to three mismatches between positions $12^{\text {th }}-15^{\text {th }}$. 4) No more than two consecutive mismatches were allowed [25]. Although homology based computational methods can cross species to identify conserved miRNA molecules, they fail to pinpoint rather unknown sequences. Having said this, due to advantages those methods can offer they have gained more popularity in recent years. Low cost and being capable of determining low abundant miRNAs as well as recognition of their temporal and spatial expression patterns are amongst some of their benefits.

\section{Results and Discussion}

\section{Identification of $A$. trichopoda potential miRNAs}

Homology-based analyses were conducted by comparing $A$. trichopoda miRNAs presented as EST (26382) and GSS (72160) deposited in GenBank. miRNAs were identified according to the procedure depicted in Figure 1. Redundant sequences were removed from the data set. The remaining sequences were subjected to secondary structure prediction by MFOLD and inspected manually against filtering criteria as indicated to check for any discrepancies. The MFEI value, gold standard in differentiating miRNAs from other small RNAs, was established [35]. All found A.trichopoda sequences were in miRNA gene families. The miRNA gene families may give rise to mature miRNAs with one or more (up to 4) different nucleotides. Newly identified miRNA from A. trichopoda EST and GSS were named by the procedure of miRNA nomenclature proposed by miRBase [28].

\section{Identification of miRNAs via EST search}

Potential miRNA genes (5) were detected in A. trichopoda ESTs (Table 1). These miRNAs fell in 5 different families. The length of identified precursor and mature miRNAs ranged from 61-192 nt (with an average $99.4 \mathrm{nt}$ ) and 19-21 nt, respectively. Despite the differences in precursor sequence length noted here, found miRNAs were predicted

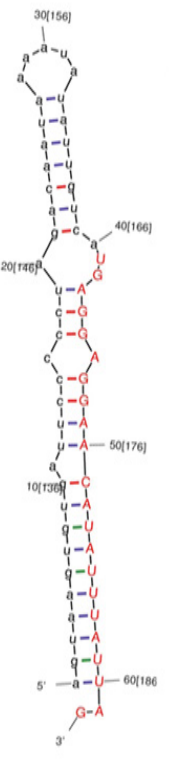

atr-miR1046

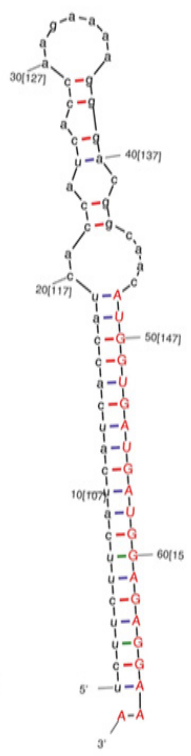

atr-miR2673
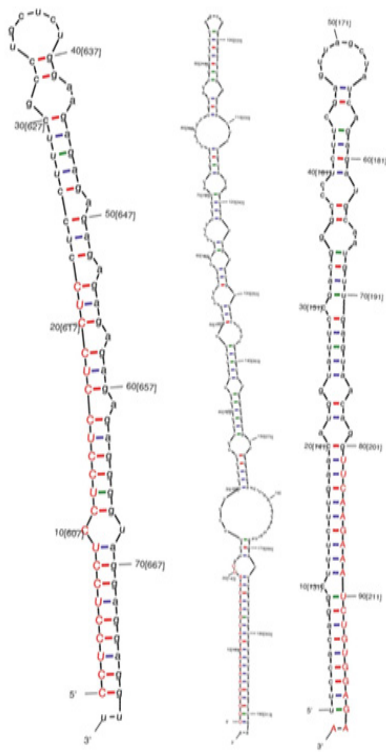

atr-miR396
atr-miR5658 atr-miR5523

Figure 2: pre-miRNA fold-back structures of $A$. trichopoda representative miRNAs found from ESTs determined by the RNA Fold MFOLD program. 
Citation: Hajieghrari B, Farrokhi N, Goliaei B, Kavousi K (2015) Computational Identification, Characterization and Analysis of Conserved miRNAs and their Targets in Amborella Trichopoda. J Data Mining Genomics Proteomics 6: 168. doi:10.4172/2153-0602.1000168

to fold into secondary structure (Figure 2; Supplementary file 1). Amongst the predicted miRNAs from ESTs, three of which are being started with uracil (Table 1 (Included as supplementary data)) and three are resided on 3' arm of the corresponding pre-miRNA secondary structure. The percentage of GC content was $28.49-59.49 \%$ with an average of $42.162 \%$. MFE and MFEI values of each identified miRNAs are shown in Table 1. Comparative analysis of A.trichopoda miRNAs with other known plants revealed two orthologs for atr- miR1046 in Physcomitrella patens, two orthologs for atr-miR2673 in Medicago truncatula, one ortholog for atr-miR5658 in Arabidopsis thaliana, and one ortholog for atr-miR5523 in Oryzae sativa. The newly identified atr-miR396f also belonged to a highly conserved miRNA family with several members identified previously in A.trichopoda as well as wide range of other plant groups from closely to distantly related species. Here, identified miRNA gene sequences used as query in Repeat Masker web based tool for classifying the miRNAs in TE-like and nonTE-like miRNA genes. The results were illustrative of atr-miR2673 and atr-miR369f genes that had simple sequence repeats in their sequences (Table 3 (Included as supplementary data)).

\section{Identification of conserved miRNAs by searching in GSSs}

Using miRNA homology-based GSS analysis, following sets of strict criteria, total of 89 conserved miRNAs were detected in A.trichopoda that were classified into 36 families (Table 2, Supplementary table S1).
Here it needs to be emphasized that GSSs are genomic in origin and therefore the predicted miRNAs require to be considered tentatively. However, the homology and structural data indicate that most derived pre-miRNA-like sequences from GSSs are genuine pre-miRNAs. These putative miRNA sequences may have the potential to be transcribed at various developmental stages of cell. Having said this, samples need to be prepared at different temporal and spatial stages or in response to biotic and abiotic stresses to determine if they are true miRNAs. Nevertheless, such global analyses of miRNA transcripts are costeffective and time consuming and bioinformatics predictions may shed some light in targeted identification of miRNAs.

GSSs predicted miRNAs were diverse in structure (Supplementary file 2) and size, even if they were from the same family. Moreover, the distribution of GSSs identified miRNAs in each family was different (Figure 3 (Included as supplementary data)). In miR5057 family, although predicted atr-miR5057a-1 and atr-miR5057a-2 as well as atr-miR5057d-1 and atr-miR5057d-2 had the same precursor length and fold back secondary structure, their genomic positions were quite different.This can be explained either by miRNA duplications or via clone overlap. In contrast, other members of the predicted atr-miR5057familyhad different precursor miRNA sequence length with different fold back secondary structures that predicted to be in different genomic positions. Meanwhile, atr-miR5057f and atrmiR5057g were originated from both sense and antisense strands of

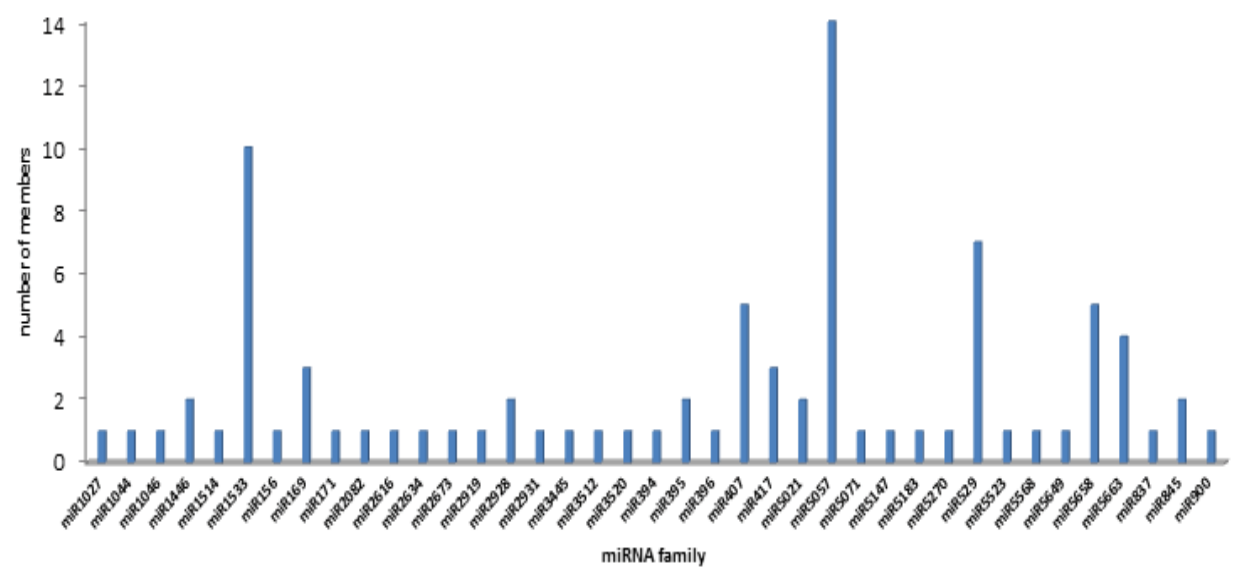

Figure 3: Number of miRNAs identified in 39 miRNA families in A.trichopoda GSSs and ESTs.

>input_sequence_36443-MINUS (gi|316086356|gb|HR647835.1/HR647835 AT_SBa0044C21.t AT__SBa Amborella trichopoda genom ic clone
AT_SBa0044C $215^{\circ}$, genom ic survey sequence)

GGGAUUUCGCCUACCCUCUUCCACGUCCAAAGUUCAUCGGACGGUUACGAUUAUCCGGUAGCAUUUC AUUUUUCAUUUUUUGUCUAUAAA(mi R5057)U (miR5568) GGAAUG ACUUAAAAUUUGGA AC GUGUGUUC AUCAAAAAUAG GUGAAUCUAC AAAAAUAUC AAAAAC AUAUUUU AC AUG AAA AAAAUUGACCAAUAUACCCCUCAAAAGUUUUGGAGGGUAUUUUGGUCAAUUUGUCUUUUCACGAAUUAUUUUUUUUAGGUGCGCCUCACUAAG AUGUACACACGCUCCAAAUUUCAAAUCAUGCUGAUU ( $S T O P$ )UAUAGAAAAAAAAAAUGAAAAAUGAAAC ACUAC CGGAC AAUCGUAACUGUGGGAU GAACUUUGGACAGUGAUGAAGGUAAGGUGAAAUCACUGUUC AUUUUCGCCUAUAGGCGUUGGGAAGACUUCUCCUAUAUAUAUAUAUAUAUGA GUGAUUUGAUGCAAUAUUUGUGUAGUCAAU AUAAUGAAAC AAU AUACGAUCUAUUAUCUUGAC CUUUC AUUCUUUC AAUAAAGGCCUUAGGCU CCAC C C AAAAGAAGGAAAUAG GGUCGAUU CUGGUUAGAC CUUUG GUUUGGC CAAC AAACUCUC AAAG AAC GGUUCCC AAAAUUAUUCUCAUUG GAGAGAAACCAAGGAGCAACUGUUUCAGAGGAAAUGAUCGAGACAGAAGCUGUCUGAUCAUGGCACCCCAAUUUCAGGAGAGACCUUUCUGAU GAAGAGAUUACUCUUUUCUCAAAC CUCUCUCAUUCUCUCUC CUUUUGUUUCCUAGCUCUUCAAGGUCAGGAC AGGUUGGUUUGGACUCCCUC UGCCUCAAGAACUUUCUCGGUCUCAUCCUUCUACUUGUUUCACUCUCAACCCCCAACCUUCCCUUUAAAUCCGACCUCCCGAGUGGCG

Figure 4: GSS (gi|316086356|gb|HR647835.1|HR647835) containing the overlap miRNA encoded within the same location. Underlined red-colored sequence represents the precursor atr-miR5568 and red-colored sequence represents the precursor atr-miR5057h. Green highlighted sequence represents mature atr-miR5568 and yellow highlighted sequence represents mature atr-miR5057h. 
Citation: Hajieghrari B, Farrokhi N, Goliaei B, Kavousi K (2015) Computational Identification, Characterization and Analysis of Conserved miRNAs and their Targets in Amborella Trichopoda. J Data Mining Genomics Proteomics 6: 168. doi:10.4172/2153-0602.1000168

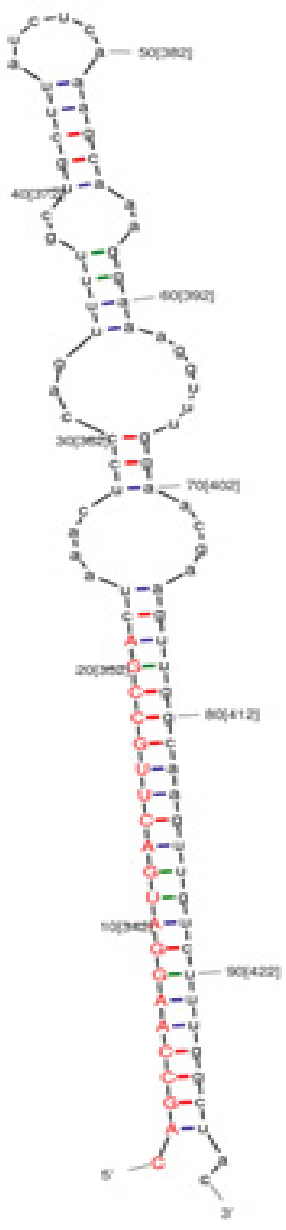

atr-miR169d-1

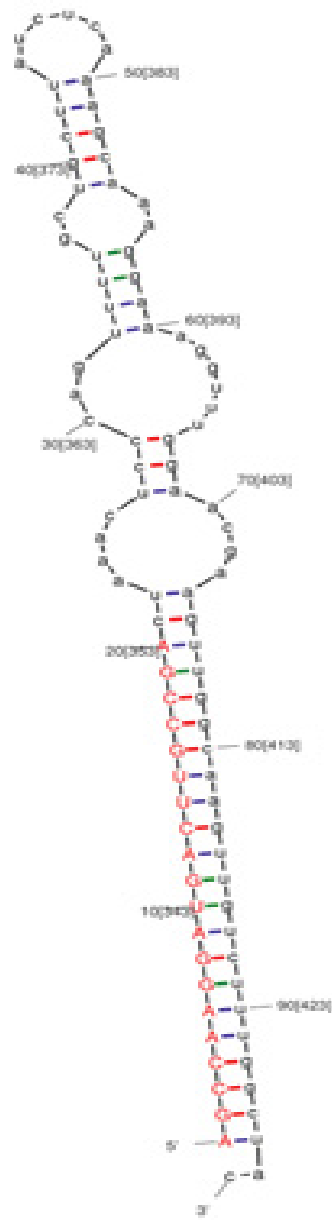

atr-miR169e

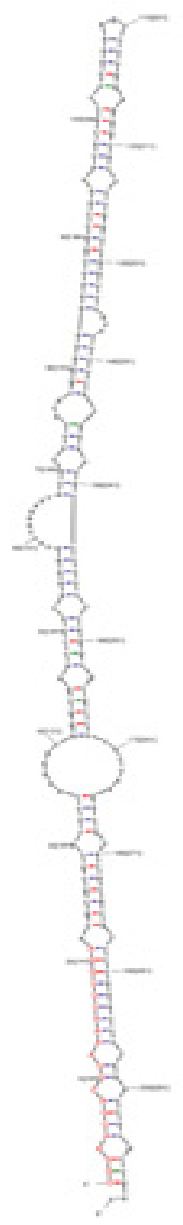

atr-miRss6s

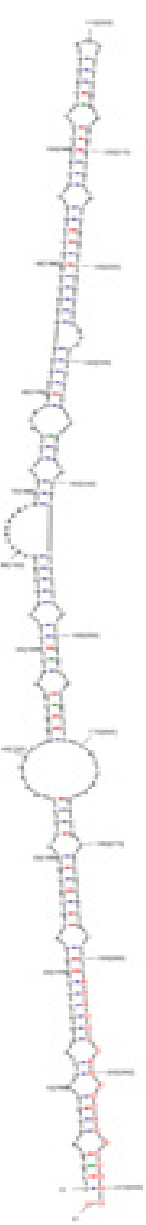

atr-miR5057

Figure 5: Predicted stem-loop hairpin secondary structure of the identified overlapped miRNAs in gi|316147995|gb|HR676573.1|HR676573 (left) and gi|31608635 6|gb|HR647835.1|HR647835 (right) submitted sequences in A. trichopoda GSS database respectively. These secondary structures were generated using MFOLD algorithm

>input_sequence_63131-PLUS (gi|316147995 |gb|HR676573.1 |HR676573 AT_SBa0083G22.r AT__SBa Amborella trichopoda genomic clone AT_SBa0083G22 3', genomic survey sequence) TGTTTCTTATATGTTITGGAGAGAGAGAGACCTTATCACTTCCTCTTTATTCTATTGGGCCAAACATTAGAT ACTTCTAGGACCTGTCCTCATCTCCTCGACTGAAGATAATGCATAATGCAATTTCTGCCATACCATCTTAAC ACCCCACTATITTCAGCCTCTCACTATCAGAACTCTATATAAACACCCCAAATATCTCTCTCCCCTTCAATGC TCTCCTCTCAGTCTCTTCCCCCCAACTCACACATCTCTCTCTCTCTCTCTCTCTCTATGGCAAAACTACTCTCT CTCATCTATGGCTCTGGCATGCAGGTTCAGAAGGCATAATG(start)C(start)AGCCAAGGATGACTTGCCG ACTAAACTCCCAGTITTGCTGCTTATCTCAAAGCAAAGGAAAGGTITGGAACGAAGTTGGCAAGTTGTCT TTGGCTAC(stop,stop)ATAATTACATTCTGATTTCTCATGCCAGGTCCATGATGAAAACAATGCACACACA CCCCATCATGTAGCACCTCAAGGAAGAAAATCAGCTTCTCTTCTTCATTAAACATGGTAATCTTCTCCCTCT ATTTCCTATCCACTCATATAATAACTTCCACCAAATGCATTATCGTAATAGTTAAATCTTTGATA

Figure 6: GSS (gi|316147995|gb|HR676573.1|HR676573) containing the overlap miRNA encoded within the same location. Red colored sequences represent the precursor sequences. Yellow highlighted sequence represent atr-miR169d-1 mature sequences and red colored underline sequences represent atrmiR5169e mature sequence. 
Citation: Hajieghrari B, Farrokhi N, Goliaei B, Kavousi K (2015) Computational Identification, Characterization and Analysis of Conserved miRNAs and their Targets in Amborella Trichopoda. J Data Mining Genomics Proteomics 6: 168. doi:10.4172/2153-0602.1000168

the miRNA genomic loci with different precursor miRNA sequence. Both sense and antisense miRNAs seems to be transcribed from the same genomic locus; however the pri-miRNAs of both strands are separately transcribed from their own DNA template strands. Thus, they may be involved in different functions in plants [36]. Here, three pairs of other miRNAs in sense/antisense strands belonging to miR1533 (atr-miR1533f and atr-miR1533g), miR845 (atr miR845a and atr-miR845b: with one ortholog in Physcomitrella patens) and miR2928 (atr-mir2928a and atr-miR2928b: with one ortholog in Oryzae sativa) families were identified that appeared to have only one pair of sense/ antisense miRNA in their families.

Newly identified atr-miR5057g and atr-miR5568 are produced from the same precursor in the same direction on the same genomic locus (gi|316086356|gb|HR647835.1|HR647835; Figures 4,5). These socalled overlapped miRNAs are transcribed from the same pri-miRNA, but they may regulate different gene(s) similar to clustered miRNAs. Another overlapped miRNAs were also identified in miRNA196 family, which included 2 miRNAs belonging to the newly identified atr-miR169d-1 and atr-miR169e with respect to a single nucleotide difference of their precursor and mature miRNA sequences on the same genomic locus (gi|316147995|gb|HR676573.1|HR676573; Figures $5,6)$. In contrast with animals that miRNA clusters have been identified widely, little reports are available for clustered miRNAs in plants [3738]. Occurrence of miRNA clusters has been reported previously in the miR169 family $[36,39]$. However in this study we did not found miRNA clusters in miR169 family. Since the miR169 family has been observed in distantly related plant species, it can be said that it is amongst the most conserved miRNA families. We also identified a member of conserved miR171 family (atr-miR171d) that had three previously identified paralogs in A.trichopoda, a member of miR165 family (atr-miR165), a member of miR394 family (atr-miR394) and two members of miR395 family (atr-miR395b and atr-miR395c). These family members had several orthologs based on search at PMRD (Plant microRNA database) [40].

Moreover, newly conserved miRNAs (10) from miR1533 family were identified. In this family, 4 paralogs of atr-miR1533e were identified that had the same precursor sequence and secondary hairpin structure. For this family, only one ortholog (gma-miR1533) has reported in Glycine max [41].

Another identified miRNA in A.trichopoda was miR529 family with 6 paralogs and several orthologs using PMRD. Meanwhile, 4 paralogs in A.trichopoda for miR5658 family were identified with an ortholog in A. thaliana reported in PMRD. Ad infinitum, 5 newly conserved miRNA genes were identified belonging to miR407 family with 3 orthologs in A. thaliana [42], Zea mays [43] and Gossypium hirsutum [44].

A miRNA gene may rise from multiple locations due to identical sequences of pre-miRNAs.For instance, miRNAs were reported for miR417 in three copies in A. trichopoda with two orthologs in A. thaliana and Oryzae sativa. Furthermore, for miR5663 family 2 miRNAs were identified (atr-miR5663a, atr-miR5663b), which atr-miR5663a had 3 copies with one ortholog in A. thaliana [45]. Other newly identified miRNAs in A. trichopoda were atr-miR1027and atr-miR1024, each with two previously identified paralogs in Physcomitrella patens. Moreover, atr-miR1446-1 and atr-miR1446-2, two identical copies of a sole miRNA appearing in different genome positions, have 5 orthologs in Populoustrichocarpa [46]. atr-miR1514 had 2 orthologs in Glycine max and one in Phaseolus vulgaris. atr-miR2082, atr-miR2616,atrmiR2634, atr-miR2919, atr-miR2931 and atr-miR3445each had one ortholog in P. patens, Medicago truncatula, O. sativa, and A. thaliana, respectively. atr-miR3512 and atr-miR3520 were also identified, each with two orthologs in Arachis hypogaea. Last, atr-miR5021aand miR5021b from miR5021 family had one ortholog in A. thaliana based on miRNAs registered in PMRD database for these species. atr-miR5071(with one ortholog in O. sativa registered in PMRD), atr-miR5147(with one ortholog in O. sativa), atr-miR5183(with one ortholog in Brachypodium distachyon registered in PMRD), atrmiR5270(with two orthologs in Medicago trunctum registered in PMRD), atr-miR5568(with one ortholog in Sorghum bicolor registered in PMRD), atr-miR5649(with two orthologs in A. thaliana registered in PMRD) and atr-miR900(with one ortholog in P. patens registered in PMRD) were identified by homology search in GSSs of $A$. trichopoda. The $\mathrm{G}+\mathrm{C}$ contents of the predicted pre-miRNA sequences were $25.09 \%$ to $50.66 \%$ with an average of $33.84 \%$. Mature miRNA sequences 16 out of 82 were started with uracil. Meanwhile, for 82 identified miRNAs, $51.22 \%$ were found to be located on the 5 ' arms of the stem loop hairpin while the rest (48.78\%) were on the $3^{\prime}$ arm (Table 2).

\section{Conservation analysis in predicted miRNA families}

Some miRNA families exist broadly in plant species and several miRNA families have multiple members within the same plant species. Different size of miRNA precursors usually results in a slightly different secondary stem-loop hairpin structure; this structure is often conserved within the same family. In this study, secondary structure constructive and structural alignment of the miR1533, miR5057, miR5663, miR529 and miR407 genes were built. Aligned precursors in each family showed some sequence similarity within their paralogs (Figure 7), but continuous conserved regions were not the same in the families. For miR5057 and miR407 families, paralogous sequences demonstrated to have stringent conservation patterns, as shown in peak of conservation (Figure 7).

Plant miRNA genes with high sequence similarity within their precursors are more likely derived from the same gene families. In these paralogs, based on duplication-mutation scenario, the gene expansion seems to have recent origin and this evolutionary pattern is probably an ongoing event. Diverged members of the same miRNA families may have been evolved at different rates within the same plant species and therefore they differ widely within and between species. Different regions in miRNA genes also seem to be under different evolutionary pressures, with higher level of conservation for the vital parts for processing and function as one expects. A growing body of evidence suggests that the mature miRNA sequence (preserved for both maintenance of the double strands and miRNA-target interactions) and its complementary sequence in the opposite arm of the fold back structure of pre-miRNA are the conserved regions [20]. While, other parts of miRNA precursors differ greatly. Here, multiple sequence alignment with a consensus structure of the families showed that the similarities not only lie on the mature $\mathrm{miRNA} / \mathrm{miRNA}^{\star}$ regions, but also throughout the genes (Figure 7). Loss of similarity may be due to miRNA locus age [47]. Accordingly, it would be fair to state that the loci more likely have been generated by duplication of pre-existing miRNA genes in the same family. Moreover, they may share a common ancestry as proposed previously for several plant miRNA families [48]. Interestingly, little gene duplication was noted for some miRNA families; for instance in miR5057 and miR529 that they have 2 copies, and miR1533 has 4 copies. Phylogenetic analysis of the precursor miRNAparalogs in these families also provided additional evidence about the origin of the duplicated loci (Figure 8). In miR5057 family, atr-MIR5057a had the same origin with other paralog supporting a probable recent duplication event. This evidence also was slightly seen in the miR529 and miR5663 families. Thus, the origin of some miRNA 
Citation: Hajieghrari B, Farrokhi N, Goliaei B, Kavousi K (2015) Computational Identification, Characterization and Analysis of Conserved miRNAs and their Targets in Amborella Trichopoda. J Data Mining Genomics Proteomics 6: 168. doi:10.4172/2153-0602.1000168

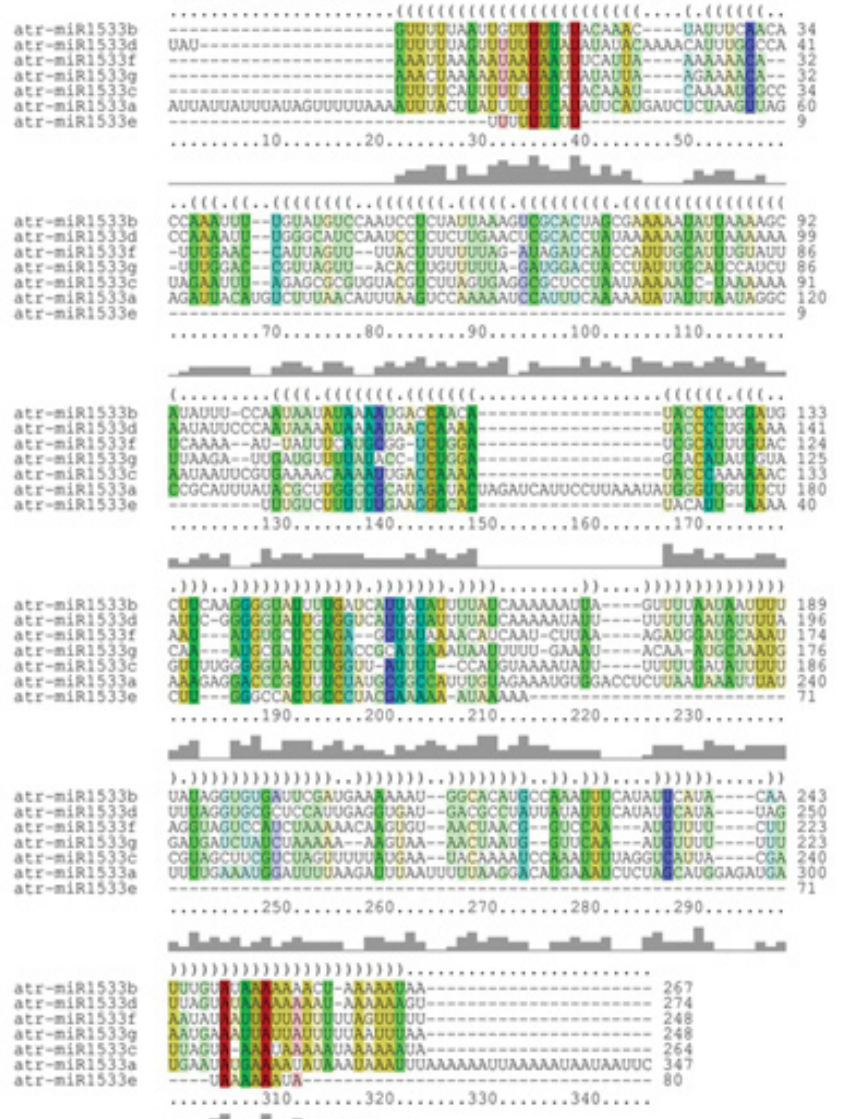

A

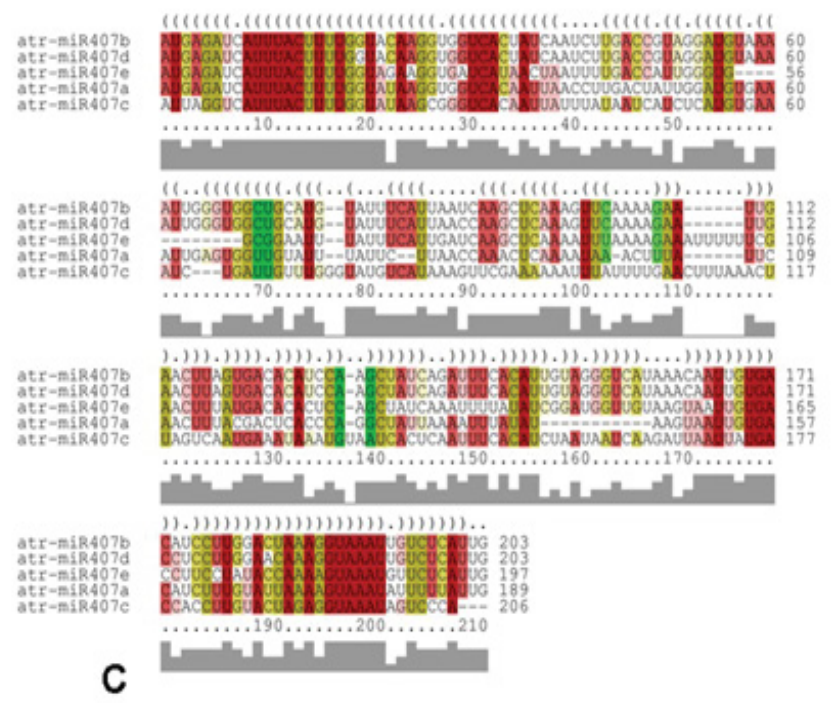

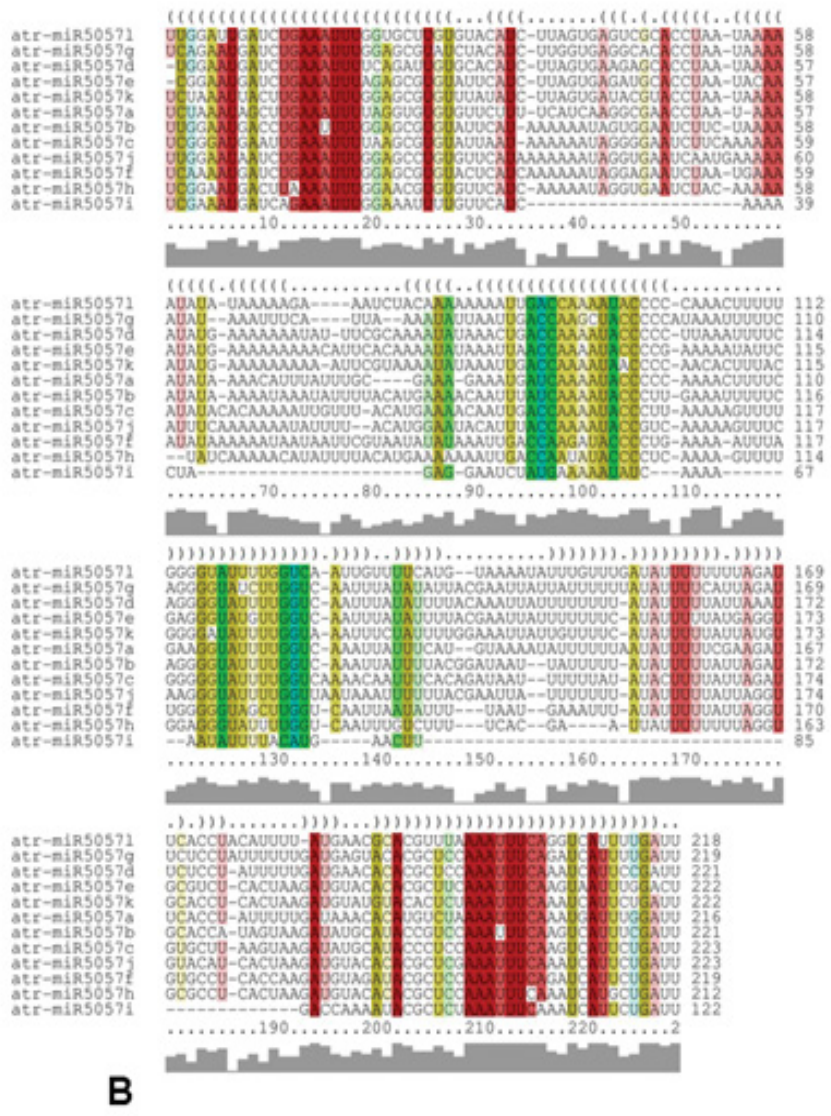

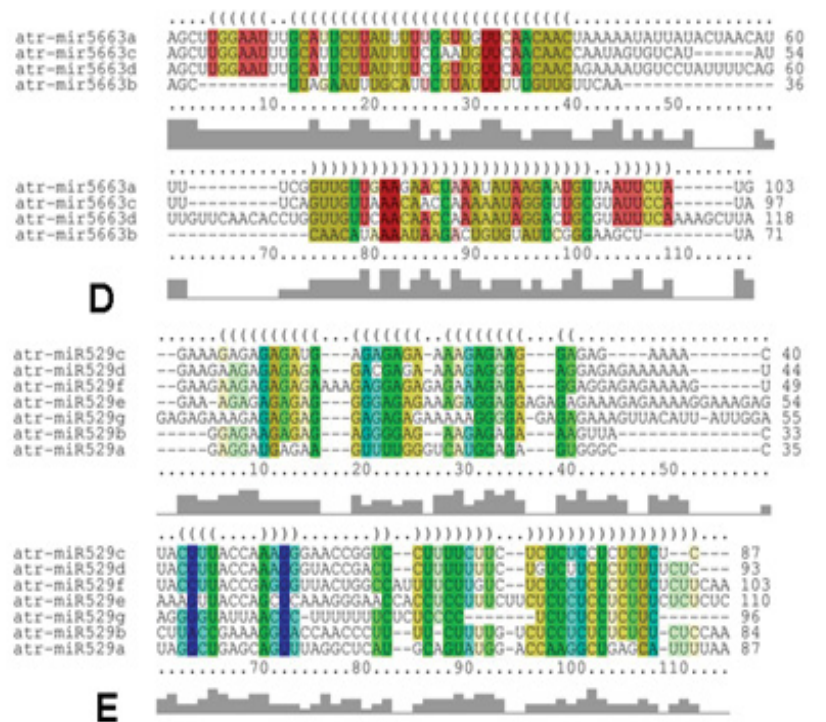

Figure 7: structural multiple sequence alignment of the precursor sequences of the newly and previously identified miRNAs in Amborella trichopoda in some conserved families using web-based computer software LocARNA. A: miR1533 family, B: miR5663family, C: miR529 family, D: miR407 family and E: miR5057 family. 
Citation: Hajieghrari B, Farrokhi N, Goliaei B, Kavousi K (2015) Computational Identification, Characterization and Analysis of Conserved miRNAs and their Targets in Amborella Trichopoda. J Data Mining Genomics Proteomics 6: 168. doi:10.4172/2153-0602.1000168
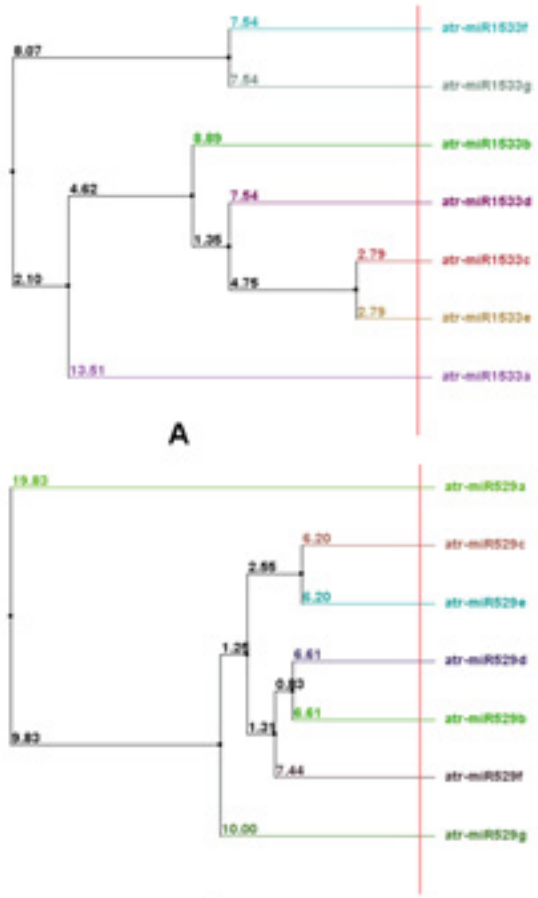

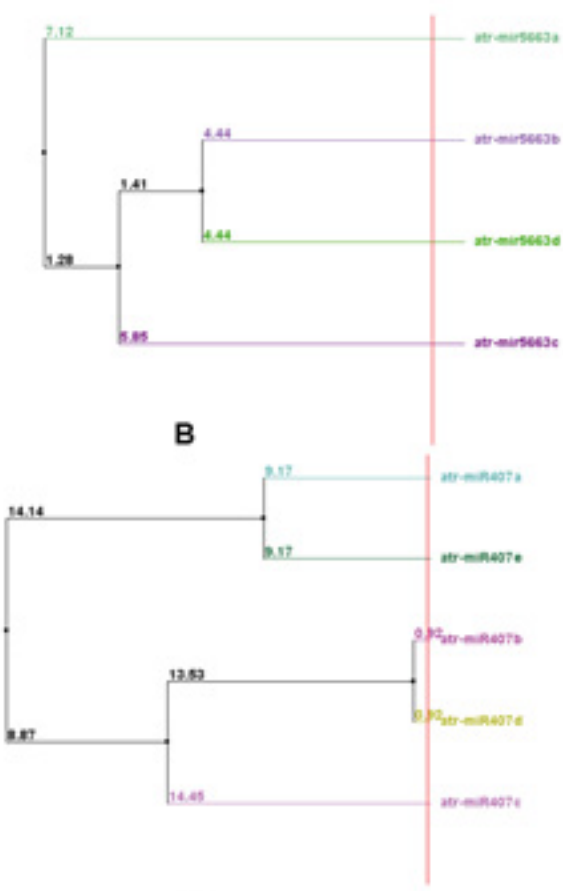

D

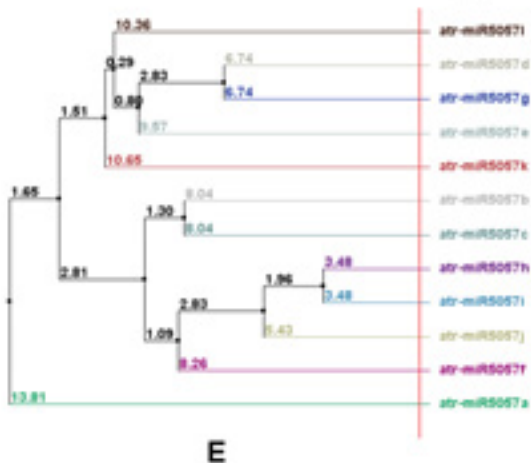

Figure 8: Phylogenetic tree obtained by aligning the precursor sequences of the newly and previously identified conserved miRNAs in A. trichopoda in some conserved families. The tree was constructed based on average distance using percent identity. A: miR1533 family, B: miR5663family, C: miR529 family, D: miR407 family and E: miR5057 families.

genes could be explained by duplication-mutation events whereby miRNA may evolve by duplication of a pre-existing miRNA.

\section{Prediction of targets for identified miRNAs}

Identification of targets for identified miRNAs is an important step for understanding the role of miRNAs and their various cellular functions via gene regulatory networks. As explained by various studies, plant miRNAs bind to the protein-coding regions of their mRNA targets with perfect or near perfect sequence complementarities, regulating gene expression by either cleavage of mRNA into two pieces or repression of translation [49]. This concept allows searching for target messengers via homology search approach. Here, miRNAs of A.trichopoda were subjected as query in psRNA Target against scaffold assignment of A.trichopoda genome sequence downloaded from Plant Ensemble. Totally, 1219 scaffold positions were identified in A. trichopoda genome. Amongst the relevant miRNA genes, 941 (77.194\%) were predicted to be subjected to mRNA cleavage. The rest (278 scaffolds) seem to be regulated via translational repression such as atr-miR3512 and atr-miR1044 (Supplementary Table S2). Based on the results, many miRNAs such as miR2082, miR2634 and miR529 families regulate several different positions on the A. trichopoda genome. Activities of the large number of identified scaffolds were also seen to be targeted by multiple miRNAs, similar to other recent studies $[23,25]$. Therefore regulatory function of miRNAs in biological and metabolic processes should be focused more and more in network concepts instead of individual connections between miRNA and its targets.

On the other hand, for some predicted miRNAs no target sequence was determined. No target miRNAs in A. trichopoda scaffolds were atr-miR1533a, atr-miR1533e, atr-miR1533f, atr-miR1533g, atrmiR2919, atr-miR407c, atr-miR407d, atr-miR407e, atr-miR417a, atrmiR5057d, atr-miR5057k, atr-miR5057l, atr-miR147, atr-miR5663c, atr-miR5663d, atr-miR845a, atr-miR900a and atr-miR2673. Lack of targets for predicted miRNAs was also reported in previous studies $[23,25]$. Targets-less miRNAs may target some positions in the genome 
Citation: Hajieghrari B, Farrokhi N, Goliaei B, Kavousi K (2015) Computational Identification, Characterization and Analysis of Conserved miRNAs and their Targets in Amborella Trichopoda. J Data Mining Genomics Proteomics 6: 168. doi:10.4172/2153-0602.1000168

Page 9 of 10

of invasive pathogens such as viroid and/or viruses that inject their genomes into the host plant [50,51]. Moreover and most likely, through evolutionary forces, genomes may have lost their corresponding target sites.Hundreds of thousands of hairpin structure in a genome exist that many of them appear to be derived from repeats, transposable elements and transposable inverted repeats as well as other evolving mechanisms that have the potential to form extremely stable miRNA like hairpin structure. It seems that newly emerged target-less miRNA is functionally inconsequential. Additionally, they are evolving much faster than that of so-called targeted miRNAs to generate seed sequences by chance. However, one needs to keep in mind that the genomic sequences that were taught are junk at one time, are not junk anymore. Accordingly, these non-specific newly emerged miRNAs may have unknown functions such as binding to some target sites (protein/nucleic acid) via an intermediate molecule(s) orget lost. The latter may be either an indication of the loss of target genes during the course of evolution or may be due to the activity of jumping genes. From earlier studies in maize by Barbara Mc-Clintock [52], it is evident that some transposons jump in a gene and once they leave, some parts of the gene that they have resided in get stuck to the transposable element and transfers to somewhere else in the genome. These movements may lead to the loss of target sequences for previously evolved miRNAs. Similar cases have mainly reported in animals [53]. In our study, similar cases were noted (TE Table 3). From the target-less miRNAs only atr-miR1533a and atrmiR2673 genes contained simple repeat elements in their sequences and classified in TE-like miRNAs. However, from this class of miRNAs we identified miRNAs such as atr-miR396f that targeted some scaffold positions on A. trichopoda draft genome (Supplementary Table S2).

\section{Conclusion}

During the past few years, with the advent in availability of sequence resources in databases as well as computational based miRNA identification tools, considerable efforts have been made to predict new miRNAs by sequence and structure homologies search in ESTs and GSSs as well as identifying miRNA targets. In the present study, we performed ESTs and GSSs based-homology search to identify A. trichopoda potential miRNAs and their targets. Our data confirmed 5 miRNA genes identified from ESTs as well as 82 potential miRNA genes from GSSs belonging to 5 and 39 miRNA gene families that are homologs to previously deposited miRNAs in public miRNA databases. Also in this study we searched for the identified miRNAs target sites in A. trichopoda genome. We have found many miRNAs with no target site in A. trichopoda genome. Identifying miRNA and their target transcripts would be useful for other research concerned with the function and regulatory mechanisms of A.trichopoda miRNAs and will improve our knowledge about the miRNA mediated mechanisms regulating plant growth and development. Understanding miRNAs, their structures and target sites may deliver greater promise towards designing and engineering new miRNA molecules. Consequently, these newly developed miRNAs may be used in our task towards improving plant resistance/tolerance to biotic and abiotic stresses, eventually improving yield and well-being of crops.

\section{References}

1. Zhang B Pan X, Cobb GP, Anderson TA (2006) Plant microRNA: a small regulatory molecule with big impact. Dev Biol 289: 3-16.

2. Berezikov E1 (2011) Evolution of microRNA diversity and regulation in animals. Nat Rev Genet 12: 846-860.

3. Yang T Xue L, An L (2007) Functional diversity of miRNA plants. Plant Science 172: 42432.

4. Xie Z Allen E, Fahlgren N, Calamar A, Givan SA, et al. (2005) Expression of
Arabidopsis MIRNA genes. Plant Physiol 138: 2145-2154.

5. Bartel DP1 (2004) MicroRNAs: genomics, biogenesis, mechanism, and function. Cell 116: 281-297.

6. Zhang Y1 (2005) miRU: an automated plant miRNA target prediction server Nucleic Acids Res 33: W701-704.

7. Pashkovskiy PP Ryazansky SS (2013) Biogenesis, evolution, and functions of plant microRNAs. Biochemistry (Mosc) 78: 627-637.

8. Dezulian T Remmert M, Palatnik JF, Weigel D, Huson DH (2006) Identification of plant microRNA homologs. Bioinformatics 22: 359-360.

9. Chen X1 (2005) MicroRNA biogenesis and function in plants. FEBS Lett 579 5923-5931.

10. Bollman KM Aukerman MJ, Park MY, Hunter C, Berardini TZ, et al. (2003) HASTY, the Arabidopsis ortholog of exportin 5/MSN5, regulates phase change and morphogenesis. Development 130: 1493-1504.

11. Park MY Wu G, Gonzalez-Sulser A, Vaucheret H, Poethig RS (2005) Nuclear processing and export of microRNAs in Arabidopsis. Proc Natl Acad Sci U S A 102: 3691-3696.

12. Voinnet $O$ (2009) Origin, biogenesis, and activity of plant microRNAs. Cell 136: 669-687.

13. Hammond SM Bernstein E, Beach D, Hannon GJ (2000) An RNA-directed nuclease mediates post-transcriptional gene silencing in Drosophila cells. Nature 404: 293-296.

14. Krek A Grün D, Poy MN, Wolf R, Rosenberg L, et al. (2005) Combinatorial microRNA target predictions. Nat Genet 37: 495-500.

15. Oliveira JS, Mendes ND, Carocha V, Craca C, Paiva JA, et al. (2013) A computational approach for micro RNA identification in plants: combining genome-based predictions with RNA-seq data. J Data Mining Genomics proteomics 4: 130 .

16. Jones-Rhoades MW Bartel DP (2004) Computational identification of plant microRNAs and their targets, including a stress-induced miRNA. Mol Cell 14: 787-799.

17. Zhang BH Pan XP, Cox SB, Cobb GP, Anderson TA (2006) Evidence that miRNAs are different from other RNAs. Cell Mol Life Sci 63: 246-254.

18. Ng Kwang Loong S Mishra SK (2007) Unique folding of precursor microRNAs: quantitative evidence and implications for de novo identification. RNA 13: 170 187.

19. Yin ZJ Shen FF (2010) Identification and characterization of conserved microRNAs and their target genes in wheat (Triticum aestivum). Genet Mol Res 9: 1186-1196.

20. Zhang B Pan X, Cannon CH, Cobb GP, Anderson TA (2006) Conservation and divergence of plant microRNA genes. Plant J 46: 243-259.

21. Li Y Li W, Jin YX (2005) Computational identification of novel family members of microRNA genes in Arabidopsis thaliana and Oryza sativa. Acta Biochim Biophys Sin (Shanghai) 37: 75-87.

22. Wang X Zhang J, Li F, Gu J, He T, et al. (2005) MicroRNA identification based on sequence and structure alignment. Bioinformatics 21: 3610-3614.

23. Das A, Mondal TK (2010) Computational identification of conserved micro RNA and their targets in Tea (Camellia sinensis). American Journal of Plant Science 1: $77-86$.

24. Galli V Guzman F, de Oliveira LF, Loss-Morais G, Körbes AP, et al. (2014) Identifying microRNAs and transcript targets in Jatropha seeds. PLoS One 9: e83727.

25. Han J Xie H, Kong ML, Sun QP, Li RZ, et al. (2014) Computational identification of miRNAs and their targets in Phaseolus vulgaris. Genet Mol Res 13: 310322.

26. Amborella Genome Project (2013) The Amborella genome and the evolution of flowering plants. Science 342: 1241089.

27. Griffiths-Jones S (2004) The microRNA Registry. Nucleic Acids Res 32: D109 111

28. Griffiths-Jones S Grocock RJ, van Dongen S, Bateman A, Enright AJ (2006) miRBase: microRNA sequences, targets and gene nomenclature. Nucleic Acids Res 34: D140-144. 
Citation: Hajieghrari B, Farrokhi N, Goliaei B, Kavousi K (2015) Computational Identification, Characterization and Analysis of Conserved miRNAs and their Targets in Amborella Trichopoda. J Data Mining Genomics Proteomics 6: 168. doi:10.4172/2153-0602.1000168

29. Griffiths-Jones S Saini HK, van Dongen S, Enright AJ (2008) miRBase: tools for microRNA genomics. Nucleic Acids Res 36: D154-158.

30. Altschul SF Gish W, Miller W, Myers EW, Lipman DJ (1990) Basic loca alignment search tool. J Mol Biol 215: 403-410.

31. Zuker M (2003) Mfold web server for nucleic acid folding and hybridization prediction. Nucleic Acids Res 31: 3406-3415.

32. Dai X Zhao PX (2011) psRNATarget: a plant small RNA target analysis server. Nucleic Acids Res 39: W155-159.

33. Han J Kong ML, Xie H, Sun QP, Nan ZJ, et al. (2013) Identification of miRNAs and their targets in wheat (Triticum aestivum L.) by EST analysis. Genet Mol Res 12: 3793-3805.

34. Will S Joshi T, Hofacker IL, Stadler PF, Backofen R (2012) LocARNA-P: accurate boundary prediction and improved detection of structural RNAs. RNA 18: $900-914$.

35. Lu Y Yang X (2010) Computational Identification of Novel MicroRNAs and Their Targets in Vigna unguiculata. Comp Funct Genomics .

36. Patanun $O$ Lertpanyasampatha $M$, Sojikul $P$, Viboonjun $U$, Narangajavana $J$ (2013) Computational identification of microRNAs and their targets in cassava (Manihot esculenta Crantz). Mol Biotechnol 53: 257-269.

37. Zhang B Pan X, Stellwag EJ (2008) Identification of soybean microRNAs and their targets. Planta 229: 161-182.

38. Xie F Frazier TP, Zhang B (2011) Identification, characterization and expression analysis of MicroRNAs and their targets in the potato (Solanum tuberosum). Gene 473: 8-22.

39. Dhandapani V Ramchiary N, Paul P, Kim J, Choi SH, et al. (2011) Identification of potential microRNAs and their targets in Brassica rapa L. Mol Cells 32: 21-37.

40. Zhang Z Yu J, Li D, Zhang Z, Liu F, et al. (2010) PMRD: plant microRNA database. Nucleic Acids Res 38: D806-813.
41. Subramanian S Fu Y, Sunkar R, Barbazuk WB, Zhu JK, et al. (2008) Novel and nodulation-regulated microRNAs in soybean roots. BMC Genomics 9: 160.

42. Sunkar R, Zhu JK (2004) Novel and stress-regulated microRNAs and other small RNAs from Arabidopsis. Plant Cell 16: 2001-2019.

43. Zhang B, Pan X, Anderson TA (2006) Identification of 188 conserved maize microRNAs and their targets. FEBS Lett 580: 3753-3762.

44. Qiu CX, Xie FL, Zhu YY, Guo K, Huang SQ, et al. (2007) Computationa identification of microRNAs and their targets in Gossypium hirsutum expressed sequence tags. Gene 395: 49-61.

45. Breakfield NW, Corcoran DL, Petricka JJ, Shen J, Sae-Seaw J, et al. (2012) High-resolution experimental and computational profiling of tissue-specific known and novel miRNAs in Arabidopsis. Genome Res 22: 163-176.

46. Lu S Sun YH, Chiang VL (2008) Stress-responsive microRNAs in Populus. Plant J 55: 131-151.

47. Fahlgren N, Jogdeo S, Kasschau KD, Sullivan CM, Chapman EJ, et al. (2010) MicroRNA gene evolution in Arabidopsis lyrata and Arabidopsis thaliana. Plant Cell 22: 1074-1089.

48. Nozawa M, Miura S, Nei M (2012) Origins and evolution of microRNA genes in plant species. Genome Biol Evol 4: 230-239.

49. Brodersen $P$, Voinnet $O$ (2006) The diversity of RNA silencing pathways in plants. Trends Genet 22: 268-280.

50. Pérez-Quintero AL, Neme R, Zapata A, López C (2010) Plant microRNAs and their role in defense against viruses: a bioinformatics approach. BMC Plant Biol 10: 138.

51. Maghuly F, Ramkat RC, Laimer M (2014) Virus versus host plant microRNAs: who determines the outcome of the interaction? PLoS One 9: e98263.

52. Ravindran S (2012) Barbara McClintock and the discovery of jumping genes. Proc Natl Acad Sci U S A 109: 20198-20199.

53. Shabalina SA, Koonin EV (2008) Origins and evolution of eukaryotic RNA interference. Trends Ecol Evol 23: 578-587. 